\title{
Influencia de la Eisenia foetida y de Sustratos Orgánicos como Agentes Bioestimulantes en la Biodegradación de un Suelo Contaminado con Petróleo Pesado
}

Celeste Fernández, María J. Llobregat, Henry Bastidas y Bonnie Sien

Universidad de Carabobo, Facultad de Ingeniería, Escuela de Ingeniería Química, Centro de Investigaciones Ambientales de la Universidad de Carabobo, Av. Universidad, Bárbula, Valencia, Edo. Carabobo-Venezuela.(e-mail: celefe@gmail.com, cfernand@uc.edu.ve)

\begin{abstract}
Resumen
Se estudió el proceso de biodegradación de un suelo contaminado con petróleo pesado proveniente de la faja petrolífera del Orinoco. El suelo fue sometido a tres tipos de tratamientos, empleando estiércol equino, humus líquido y ejemplares de Eisenia foetida; para lograr disminuir su nivel de contaminación. Se analizaron hidrocarburos totales de petróleo (HTP), fracciones del crudo, porcentaje de humedad relativa, potencial de acidez, conteo de aerobios mesófilos, de acuerdo a procedimientos estándares (ASTM, COVENIN, y EPA). Los resultados reportaron diferencias significativas para el tratamiento con E. foetida mientras que para el tratamiento con humus líquido no se registraron diferencias significativas en la prueba de Dunnett. Los tratamientos con $E$. foetida y estiércol equino presentaron un alto porcentaje de remoción de hidrocarburos saturados y resinas; mientras que las fracciones de aromáticos y asfaltenos no variaron significativamente en la prueba de Tukey HSD, siendo el tratamiento con E. foetida el más eficaz en la descontaminación del suelo impactado.
\end{abstract}

Palabras clave: biorremediación, Eisenia foetida, estiércol equino, humus líquido, petróleo pesado

\section{The influence of Eisenia foetida and Organic Substrates as Biostimulants Agents in the Biodegradation of Heavy Crude Contaminated Soil}

\begin{abstract}
The biodegradation of a heavy crude contaminated soil coming from the Orinoco oil belt was studied. The soil was subjected to three types of treatments using horse dung, liquid humus and samples of Eisenia foetida as bioestimulants to reduce its level of contamination. Total petroleum hydrocarbon (TPH), characterization of crude fractions, relative humidity rate, acidity potential and aerobics mesophylic count were performed, according to the standard methods (ASTM, COVENIN, and EPA). Significant differences in the treatments with the samples of $E$. foetida were found while no significant differences were observed in the treatment with liquid humus using Dunnett's test. The $E$. foetida and horse dung treatments shows a high degradation of saturated hydrocarbons and resins, whereas the asphalt and aromatic fractions did not vary significantly using the Tukey HSD test. The $E$. foetida treatment proved the most effective in the decontamination of heavy crude contaminated soil.
\end{abstract}

Keywords: bioremediation, Eisenia foetida, horse dung, liquid humus, heavy petroleum 


\section{INTRODUCCIÓN}

Los suelos contaminados con petróleo o sus derivados provenientes de refinerías, petroquímicas, terminales, estaciones de servicio, tanques de combustible, en los sectores industriales, originan alteraciones en los ecosistemas terrestres y marinos causando variaciones en sus propiedades fisicoquímicas y biológicas, alterando los procesos productivos, el hábitat y los paisajes naturales.

Una de las formas de degradar esa contaminación es a través de la población microbiana, constituyéndose en el principal proceso de descontaminación natural. Este proceso se puede acelerar y/o mejorar mediante la aplicación de tecnologías de biorremediación (Infante, 2001). El concepto de biorremediación se utiliza para describir una variedad de sistemas que utilizan organismos vivos (plantas, hongos, bacterias, entre otros), para remover, degradar o transformar compuestos orgánicos tóxicos en productos metabólicos menos tóxicos o inocuos. El objetivo común de estos sistemas consiste en la conversión o transformación de los compuestos contaminantes en agua, anhídrido carbónico u otro gas o sustancia inorgánica y masa celular o biomasa (La Grega et al., 2002). Por otra parte, el petróleo contiene diversidad de compuestos que lo hacen un sustrato ideal para evaluar el potencial degradativo de consorcios microbianos y de otros organismos vivos de interés en biorremediación como lombrices (Canals, 2005).

Además, los suelos contaminados tienen más microorganismos que los suelos no contaminados, pero su diversidad microbiana es más reducida, para bioestimularlos se puede emplear el compostaje, esta estrategia de biorremediación utiliza microorganismos aeróbicos, formando pilas de material que deben ser mezcladas y humedecidas periódicamente para promover la actividad microbiana, mediante el empleo de un agente bioestimulador (Gallego et al., 2004).

Al analizar específicamente el caso de la explotación petrolera en la faja petrolífera del Orinoco, las amplias reservas de petróleo en la zona, y la contaminación que se puede originar por ripios de perforación, crudos livianos y pesados, entre otros; se hace necesario la aplicación de diversas técnicas de saneamiento que sean efectivas para cumplir con las exigencias de la normativa ambiental en el país.

Por esta razón el objetivo del presente estudio es realizar una evaluación del proceso de biorremediación en el suelo contaminado, utilizando como agentes bioestimulantes: lombrices, humus líquido y estiércol equino, para lograr disminuir su nivel de contaminación.

Se decidió utilizar la E. foetida debido a la gran facilidad que tienen estas especies de ser cultivadas, además de ser un macroorganismo fuerte y con gran capacidad de tolerancia a los altos rangos de temperatura, humedad y potencial de acidez (Callaham et al., 2002). También, Schaefer y Filser (2007) emplearon este macroorganismo como agente bioestimulador en la degradación de crudo obteniendo excelentes resultados. Por otro lado Hubálek et al. (2007), comprobaron que la $E$. foetida es un organismo resistente que puede ser utilizado para monitorear la ecotoxicidad de suelos contaminados con crudo durante el proceso de biorremediación, mediante la evaluación de su adaptación, mortalidad y reproducción (Geissen et al., 2008).

Asimismo Monard et al. (2008), señalan que la E.foetida cambia las propiedades físicas y químicas del suelo, son eficientes ingenieras que actúan en las comunidades microbiológicas, actividad del suelo y pueden ser capaces de mineralizar los contaminantes presentes en este. Igualmente Contreras et al. (2008), afirman que la aplicación de lombrices a un suelo contaminado con petróleo es una alternativa de dextosificación segura, amigable y económica para remover hidrocarburos aromáticos policíclicos del suelo.

\section{MATERIALES Y MÉTODOS}

Se trabajó con un suelo proveniente de Morichal, estado Monagas en el rodal del módulo MPE - 3 de SINOVENSA (filial de PDVSA). En el muestreo del suelo se realizaron ploteos en forma de zig-zag y se seleccionaron dos puntos de muestreo (Morichal, punto 1 y Morichal, punto 2). El punto 1 se ubica geográficamente entre las coordenadas $50^{\circ} 97^{\prime} 62^{\prime \prime} \mathrm{E}$ y $96^{\circ} 49^{\prime} 19^{\prime \prime} \mathrm{N}$, con una 
pendiente de aproximadamente 1,3\%, la vegetación presente son pinos (Pinus caribea). El punto 2 se ubica entre las coordenadas $51^{\circ} 02^{\prime} 97^{\prime \prime} \mathrm{E}$ y $96^{\circ} 49^{\prime} 39^{\prime \prime} \mathrm{N}$, con una pendiente de $1 \%$ y la vegetación en el área es de chaparros y gramíneas del género Trachypogon. En cada punto seleccionado se consideraron dos horizontes a diferentes profundidades, el A [profundidad $(0-15) \mathrm{cm}$ ] y $B$ [profundidad $(30-45) \mathrm{cm}$ ]. Primeramente se limpió el terreno superficial sobre el punto de muestreo y se procedió a introducir un barreno recto para tomar una muestra cilíndrica no perturbada, la cual fue guardada en bolsas de plástico debidamente selladas e identificadas para su posterior traslado.

A las muestras tomadas se le determinaron las variables químicas: capacidad de intercambio catiónico $(\mathrm{CIC})$, porcentaje saturación con bases (\% SB), relación de absorción de sodio (RAS), pH, calcio $(\mathrm{Ca})$, fósforo $(\mathrm{P})$, nitrógeno total $(\mathrm{N})$, potasio $(\mathrm{K})$, sodio $(\mathrm{Na})$, magnesio $(\mathrm{Mg})$, conductividad eléctrica $(\mathrm{Ce})$, porcentaje de materia orgánica (\% MO), aluminio intercambiable $\left(\% \mathrm{Al}^{3+}{ }_{\mathrm{INT}}\right)$, y las variables físicas, porcentaje de arcillas $(\% \mathrm{~A})$, porcentaje de limo $(\% \mathrm{~L})$ y porcentaje de arena $(\% \mathrm{a})$.

Para el encalado del suelo fue necesario determinar la capacidad de saturación del suelo, donde se procedió a pesar aproximadamente $200 \mathrm{~g}$ de suelo virgen en un vaso de precipitado y se adicionó gradualmente un volumen de $1 \mathrm{~mL}$ de agua destilada hasta observar una ligera capa sobrenadante de agua en la superficie del suelo (ASTM D2216, 1998). Otro de los parámetros a determinar en el encalado es la densidad del suelo donde se empleó la metodología planteada por Casanova (2005). Se seleccionó el carbonato de calcio sólido $\left(\mathrm{CaCO}_{3}\right)$ para corregir el $\mathrm{pH}$ del suelo, el cual permite la corrección de la acidez por el intercambio de los cationes aluminio en la matriz del suelo. Una vez el suelo encalado, se dejó en reposo de 7 a 15 días, realizando monitoreos del pH hasta observar su estabilización.

Para la determinación del potencial de acidez se utilizó un pH-metro marca Oaklon modelo 510, equipado con un electrodo de referencia de calomel saturado y un indicador de membrana de vidrio sensible con referencia interna de $\mathrm{Ag} / \mathrm{AgCl}$. Una vez calibrado, se pesaron aproximadamente $5 \mathrm{~g}$ de suelo contaminado en un vaso de precipitado, se le adicionaron $25 \mathrm{~mL}$ de agua destilada (relación 1:5) según TMECC (2001), se agitó por espacio de 15 minutos, se filtró y al líquido sobrenadante se le determinó el pH.

Se utilizó el método de ensayo estándar para la determinación del contenido de humedad de suelo por masa (ASTMD2216, 1998). Se determinó la masa de una cápsula de Petri con tapa, sobre la cual se agregaron $20 \mathrm{~g}$ de suelo y se colocó en la estufa a $120^{\circ} \mathrm{C}$ por 12 horas, por gravimetría se determina el porcentaje de humedad. Así como la norma (COVENIN 902, 1987) para el recuento de aerobios mesófilos (UFC/g) por diluciones seriadas y cultivos en placas de agar.

En la caracterización de los agentes bioestimulantes se utilizaron dos tipos de sustrato, el estiércol equino y el humus líquido. A estos sustratos orgánicos se les realizó una caracterización para conocer la cantidad de macroelementos presentes en ellos como el carbono, nitrógeno y fósforo $(\mathrm{C}, \mathrm{N}, \mathrm{P})$ y el porcentaje de materia orgánica, con el fin de conocer si las relaciones de $\mathrm{C} / \mathrm{N}$ y $\mathrm{C} / \mathrm{P}$ se encontraban dentro del rango de valores óptimos (Infante, 2001), ya que estos son parámetros claves que garantizan parte del desarrollo de los microorganismos presentes en el suelo.

Para la biorremediación se pesaron $15 \mathrm{~kg}$ de suelo encalado al que se le adicionaron aproximadamente $850 \mathrm{~g}$ de crudo Merey, obteniéndose una concentración inicial de hidrocarburos totales de petróleo de $(5,58 \pm 0,02) \%$. Se utilizaron 7 recipientes rectangulares de polietileno de alta densidad $(P E-H D)(20 \times 15 \times 10) \mathrm{cm}$, distribuidos de la siguiente forma: uno con suelo contaminado (control), 3 réplicas con suelo contaminado y estiércol equino, 3 réplicas con suelo contaminado y humus líquido, y 3 réplicas con suelo contaminado, estiércol equino y 15 ejemplares de $E$. foetida.

Los hidrocarburos totales de petróleo fueron determinados según la norma propuesta por la Agencia de Protección Ambiental, method 3540c soxhlet extraction (US EPA, 1996), empleando tetrahidrofurano como solvente extractor en un equipo de soxhlet. En la obtención de las fracciones de crudo se siguió el procedimiento por determinación gravimétrica de saturados, aromáticos, resinas y asfaltenos (SARA), según la norma propuesta por la Agencia de Protección Ambiental, 
8020/8100 (US EPA, 1986) en una columna cromatográfica empacada con silice gel 60 de Merck

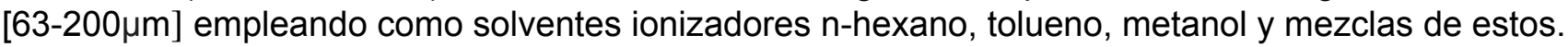

Las fracciones de resinas y asfaltenos fueron analizadas en un espectrómetro RMN- ${ }^{1} \mathrm{H}$ BRUKERAC-200. Los valores de desplazamientos químicos se obtuvieron en ppm, utilizando TMS (tetrametilsilano) como referencia interna y las muestras fueron disueltas en cloroformo. Las condiciones de registro para estos espectros fueron: Tiempo de adquisición: 2,720 s. Anchura de pulso: 5,31 ms. Tiempo entre pulsos: $1 \mathrm{~s}$.

Para realizar la comparación de las principales variables estadísticas de interés se empleó el software analítico STATISTIX 8.0 (2003) para Windows. El diseño experimental fue en bloques completos al azar. Con los resultados experimentales obtenidos se realizó un análisis de varianza (ANOVA TWO WAY). Los resultados fueron tratados mediante las pruebas de significancia cuyo valor de a predefinida se estableció igual a 0,05 (Gutiérrez y De la Vara, 2004). Se aplicaron las pruebas de estimación de supuestos, como la de Shapiro - Willk, así como también se realizó la prueba de homogeneidad de las varianzas mediante el gráfico de los residuos por valores fijos. Para lograr una comparación efectiva de los tratamientos en conjunto se realizó la prueba de Tukey HSD y para realizar la comparación de las unidades experimentales en función del tratamiento control, se empleó la prueba de Dunnett.

\section{RESULTADOS Y DISCUSIÓN}

En la aplicación de técnicas de biotratamiento es indispensable controlar la textura del suelo, la cual se refiere a las diversas clases de partículas que lo conforman (Casanova, 2005). En la tabla 1 se señalan los resultados de este análisis, de acuerdo con el triángulo textural (Casanova, 2005), la textura del suelo virgen es franco arenosa, lo cual favorece que el proceso de biorremediación se lleve a cabo de acuerdo con lo establecido en el Decreto 2635 (1998).

Tabla 1: Análisis textural del suelo virgen

\begin{tabular}{|c|c|c|c|c|}
\hline \multirow{2}{*}{ Clase textural } & \multicolumn{2}{|c|}{ Morichal punto 1 } & \multicolumn{2}{c|}{ Morichal punto 2 } \\
\cline { 2 - 5 } & Horizonte A & Horizonte B & Horizonte A & Horizonte B \\
\hline Arena $(\mathrm{Ar} \pm 0,1) \%$ & 63,0 & 65,0 & 66,0 & 67,0 \\
\hline Arcilla(Ac $\pm 0,1) \%$ & 20,8 & 24,8 & 20,8 & 20,8 \\
\hline Limo $(\mathrm{L} \pm 0,1) \%$ & 16,2 & 10,2 & 13,2 & 12,2 \\
\hline
\end{tabular}

Con respecto a los macroelementos, en la tabla 2, se muestran los valores que contribuyen al crecimiento vegetativo de las plantas en el suelo (Sandoval, 2002).

Tabla 2: Análisis de macroelementos del suelo virgen

\begin{tabular}{|l|c|c|c|c|}
\hline \multirow{2}{*}{\multicolumn{1}{|c|}{ Parámetro de interés }} & \multicolumn{2}{c|}{ Morichal punto 1 } & \multicolumn{2}{c|}{ Morichal punto 2 } \\
\cline { 2 - 5 } & Horizonte A & Horizonte B & Horizonte A & Horizonte B \\
\hline Calcio total(Ca $\pm 0,01) \mathrm{mg} / \mathrm{kg}$ & $<40,00$ & $<40,00$ & $<40,00$ & $<40,00$ \\
\hline Potasio total( $\mathrm{K} \pm 0,01) \mathrm{mg} / \mathrm{kg}$ & 270,70 & 148,89 & 87,21 & 152,41 \\
\hline Sodio total $(\mathrm{Na} \pm 0,01) \mathrm{mg} / \mathrm{kg}$ & $<40,00$ & $<40,00$ & $<40,00$ & $<40,00$ \\
\hline Magnesio total$(\mathrm{Mg} \pm 0,01) \mathrm{mg} / \mathrm{kg}$ & $<40,00$ & $<40,00$ & 57,83 & $<40,00$ \\
\hline Fósforo total( $\mathrm{P} \pm 0,01) \mathrm{mg} / \mathrm{kg}$ & 3,10 & 2,05 & $<2,00$ & $<2,00$ \\
\hline Nitrógeno total( $\mathrm{N} \pm 0,001) \%$ & 0,022 & 0,022 & 0,022 & 0,022 \\
\hline Materia orgánica(Mo $\pm 0,01) \%$ & 1,36 & 0,54 & 1,51 & 0,00 \\
\hline
\end{tabular}

Se observa que el fósforo $(P)$, en ambos horizontes, presenta valores bajos menores a $10 \mathrm{mg} / \mathrm{kg}$ lo cual indica que puede haber fijación de este elemento (Casanova, 2005). Por otro lado los resultados de calcio $(\mathrm{Ca})$, sodio $(\mathrm{Na})$ y magnesio $(\mathrm{Mg})$ son menores de $40 \mathrm{mg} / \mathrm{kg}$, esto implica que el suelo a estudiar no es propicio para el desarrollo de plantas según Sandoval (2002), en cambio los valores de potasio $(\mathrm{K})$ se encuentran en promedio superiores a $150 \mathrm{mg} / \mathrm{kg}$ lo que resulta satisfactorio 
en la fertilización (Casanova, 2005). Otro de los macroelementos es la cantidad de nitrógeno total presente en el suelo siendo esta baja, lo que limita el crecimiento de los consorcios bacterianos dentro del suelo en estudio (Canals, 2005).

Con respecto a la capacidad de intercambio catiónico el valor reportado es muy bajo, propio de suelos arenosos (ClC<10 meq/100 g de suelo) (Casanova, 2005) lo que indica que las partículas coloidales del suelo (principalmente arcillas), se encuentran saturadas por cationes aluminio $\left(\mathrm{Al}^{3+}\right)$ y protones $\left(\mathrm{H}^{+}\right)$, en lugar de cationes intercambiables como calcio, magnesio, sodio y potasio. Este tipo de suelo no tiene la habilidad de retener nutrientes como $\mathrm{Ca}$, $\mathrm{Na}$, $\mathrm{Mg}$ y $\mathrm{K}$ porque son susceptibles al lavado del perfil; esto ocasiona que el porcentaje de saturación con bases como se señala en tabla 3, sea bajo (<65\%) (Casanova, 2005), aunado a ello el decreto 2635 establece un nivel mayor a $80 \%$.

Otro de los parámetros importantes es la conductividad eléctrica del suelo que reporta un valor muy alto, mayor a $9,00 \mathrm{mmhos} / \mathrm{cm}$, lo que indica que el suelo tiene un abundante contenido de sales minerales, catalogado como potencialmente salino (Rodríguez et al., 2006). Aunado a ello, el decreto 2635 establece un máximo permitido de $3,5 \mathrm{mmhos} / \mathrm{cm}$, si se supera este límite se tiene lugar inhibición en el normal desarrollo de los cultivos.

Tabla 3: Análisis de factores abióticos de interés en el suelo virgen

\begin{tabular}{|l|c|c|c|c|c|}
\hline \multicolumn{1}{|c|}{ Parámetro de interés } & \multicolumn{2}{|c|}{ Morichal punto 1} & \multicolumn{2}{c|}{ Morichal punto 2 } & \multicolumn{2}{c|}{$\begin{array}{c}\text { Rango } \\
\text { permisible }\end{array}$} \\
\cline { 2 - 5 } & $\begin{array}{c}\text { Horizonte } \\
\text { A }\end{array}$ & $\begin{array}{c}\text { Horizonte } \\
\text { B }\end{array}$ & $\begin{array}{c}\text { Horizonte } \\
\text { A }\end{array}$ & $\begin{array}{c}\text { Horizonte } \\
\text { B }\end{array}$ & \\
\hline $\begin{array}{l}\text { Potencial de acidez } \\
\text { (pH } \pm 0,01) \text { adim }\end{array}$ & 4,55 & 4,47 & 4,28 & 4,18 & $5-8$ \\
\hline $\begin{array}{l}\text { Conductividad eléctrica } \\
\text { (Ce } \pm 0,01) \text { mmhos/cm }\end{array}$ & 31,40 & 14,77 & 17,68 & 9,76 & 3,5 \\
\hline $\begin{array}{l}\text { Saturación con bases } \\
\text { (Sb } \pm 0,01) \%\end{array}$ & 0,12 & 0,06 & 0,08 & 0,08 & 80 \\
\hline $\begin{array}{l}\text { Aluminio intercambiable } \\
\text { (A } \pm 0,001) \text { meq/100 g de suelo }\end{array}$ & 7,710 & 7,710 & 7,710 & 7,710 & 1,5 \\
\hline $\begin{array}{l}\text { Relación de absorción de sodio } \\
\text { (RAS } \pm 0,001) \text { adim }\end{array}$ & 0,128 & 0,125 & 0,050 & 0,428 & $<8$ \\
\hline $\begin{array}{l}\text { Capacidad de inter. catiónico } \\
\text { (ClC } \pm 0,01) \text { meq/100 g de suelo }\end{array}$ & 0,73 & 0,50 & 0,60 & 0,65 & --- \\
\hline
\end{tabular}

El potencial de acidez en los horizontes, es inferior a 5 y refleja acidez en el suelo, esto es debido a la presencia de altas concentraciones de iones aluminio $\left(\mathrm{Al}^{3+}\right)$ e iones hidronios, lo que conlleva a realizar la enmienda o encalado del suelo, ya que los protones liberados en el proceso de mineralización de la materia orgánica pueden reaccionar con las estructuras cristalinas de las arcillas, liberando aluminio y ácido salicílico a la disolución del suelo. El ión aluminio pasa a los sitios de intercambio de las arcillas y puede sufrir un proceso de hidrólisis produciendo una mayor cantidad de iones hidronios que disminuyen el $\mathrm{pH}$, finalmente el aluminio precipita como hidróxido de aluminio $\left[\mathrm{Al}(\mathrm{OH})_{3(\mathrm{~s})}\right]$ (Casanova, 2005).

Luego del encalado, el potencial de acidez permanece en un rango ligeramente básico, muy cercano a la neutralidad, de $(7,35 \pm 0,01)$ adim. El hecho de que el $\mathrm{pH}$ del suelo no varíe de forma significativa (variaciones mayores a la unidad), se debe principalmente a que al realizar la enmienda o encalado con carbonato de calcio éste adquirió una capacidad reguladora y es capaz de resistir los cambios bruscos de potencial de acidez. La humedad relativa se controló durante el proceso de degradación del crudo, manteniéndose aproximadamente en $5 \%$, lo que equivale a decir un valor del $60 \%$ de la capacidad de campo (Infante, 2001) para que exista el adecuado trasporte de nutrientes a través de las células de los microorganismos que ejercen la función degradadora. 
En la caracterización del petróleo el crudo Merey tiene un alto contenido de resinas $(32,9 \pm 0,8) \%$, de hidrocarburos aromáticos $(32,8 \pm 0,8) \%$, además de poseer un alto contenido de asfaltenos $(4,3 \pm 0,8) \%$ y una fracción saturada de $(29,8 \pm 0,8) \%$, catalogándolo como crudo pesado.

Con respecto a la caracterización de los agentes bioestimulantes resulta que el estiércol equino es rico en el nutrimento fósforo, en materia orgánica y en nitrógeno total, no obstante, algunos autores, como Sandoval (2002) sugieren que los estiércoles se deben utilizar más como acondicionadores y mejoradores del suelo que como fertilizantes, debido a baja cantidad de nutrientes que aportan con respecto a la excesiva demanda de éstos que tienen los suelos.

Para el alimento, sustrato, de las lombrices E. foetida se usó el excremento de caballo, estiércol equino, ya que el mismo posee un $\mathrm{pH}$ cercano a la neutralidad en estado de post-maduración. La necesidad de adicionar sustrato se debe a que estos macroorganismos no son de tierra, es decir, no son autóctonos del suelo así como tampoco son capaces de sobrevivir con los nutrientes que naturalmente posee el suelo (Ferruzzi, 1994).

El tercer bioestimulante empleado fue el humus líquido ya que posee una gran fuente de nutrimentos como nitrógeno, carbono y fósforo, así como el estiércol, sin embargo la tabla 4 muestra que los macroelementos presentes en el humus líquido son muy bajos. El humus proviene de una solución del lavado de la pila de compostaje, lo que origina que el residuo esté muy diluido, razón por la cual se observan valores tan bajos de macroelementos en este agente bioestimulante (Durán y Henríquez, 2007).

Tabla 4: Caracterización de los agentes bioestimulantes

\begin{tabular}{|l|c|c|}
\hline \multicolumn{1}{|c|}{ Parámetro } & $\begin{array}{c}\text { Estiércol } \\
\text { equino }\end{array}$ & $\begin{array}{c}\text { Humus } \\
\text { líquido }\end{array}$ \\
\hline Materia orgánica $(\mathrm{Mo} \pm 0,02) \%$ & 1,81 & 0,34 \\
\hline Carbono total $\left(\mathrm{C}_{\mathrm{T}} \pm 0,02\right) \%$ & 1,05 & 0,19 \\
\hline Nitrógeno total $\left(\mathrm{N}_{\mathrm{T}} \pm 0,02\right) \%$ & 0,14 & 0,16 \\
\hline Fósforo total $\left(\mathrm{P}_{\mathrm{T}} \pm 0,2\right) \mathrm{mg} / \mathrm{kg}$ & 290,2 & 100,0 \\
\hline
\end{tabular}

De acuerdo con Infante (2001) para que el proceso de biorremediación sea exitoso, es imprescindible mantener una relación de nutrientes adecuado, los valores están reportados en la tabla 5. Para el tratamiento control las relaciones de nutrientes indican que existe un déficit de nitrógeno y de fósforo ya que son mayores al rango óptimo, lo que sugiere que los microorganismos autóctonos del suelo no podrán degradar todo el carbono presente en el suelo debido a que hay muy pequeñas cantidades de nitrógeno y de fósforo, necesarios para el desarrollo bacteriano. En cambio con estiércol equino y la $E$. foetida, las relaciones arrojan resultados favorables dentro del rango propuesto por Levin y Gealt (1997).

Tabla 5: Relación de nutrientes en los biorreactores al inicio del tratamiento

\begin{tabular}{|c|c|c|c|c|}
\hline \multirow{2}{*}{$\begin{array}{l}\text { Tipo de } \\
\text { tratamiento }\end{array}$} & \multicolumn{2}{|c|}{$\begin{array}{l}\text { Relación de nutrientes } \\
(R \pm 0,04) \text { adim }\end{array}$} & \multicolumn{2}{|c|}{$\begin{array}{c}\text { Relación de nutrientes } \\
\text { óptima }\end{array}$} \\
\hline & $(\mathrm{C} / \mathrm{N})$ & $(\mathrm{C} / \mathrm{P})$ & $(\mathrm{C} / \mathrm{N})$ & $(\mathrm{C} / \mathrm{P})$ \\
\hline Control & 197,84 & $14.040,00$ & \multirow{4}{*}{$45-100$} & \multirow{4}{*}{$650-900$} \\
\hline Estiércol equino & 93,25 & 679,83 & & \\
\hline Humus líquido & 102,36 & $3.473,20$ & & \\
\hline E. foetida & 93,25 & 679,83 & & \\
\hline
\end{tabular}

En la determinación del HTP para cada uno de los biorreactores se empleó tetrahidrofurano $\left(\mathrm{C}_{4} \mathrm{H}_{8} \mathrm{O}\right)$ como solvente extractor, ya que es un compuesto heterocíclico moderadamente polar, lo cual permite solubilizar los diferentes tipos de compuestos presentes en el crudo al realizar el análisis SARA (Vívas et al., 2002). Los resultados señalados en la figura 1, revelan que existe una disminución de los hidrocarburos totales de petróleo en función del tiempo, esto se debe principalmente al fortalecimiento de comunidades bacterianas autóctonas que logran degradar parte 
de los compuestos que están presentes en el crudo, debido a que se mantienen ciertas condiciones que propician su desarrollo (Canals, 2005).

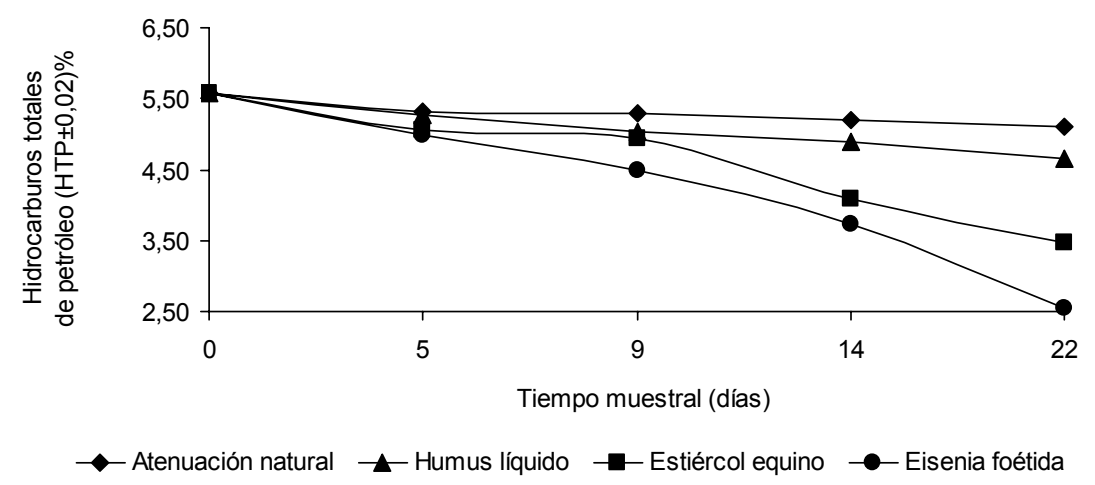

Fig. 1: Disminución de los hidrocarburos totales de petróleo en cada uno de los tratamientos

Según La Grega et al. (2002) las bacterias son las responsables de las reacciones de biodegradación y acotan que aunque dichas reacciones forman parte normal del metabolismo de una célula bacteriana, el objetivo de estos microorganismos no es la eliminación de los contaminantes ambientales; sino crecer y mantenerse. El desarrollo de la carga bacteriana mesófila se evidencia en la figura 2.

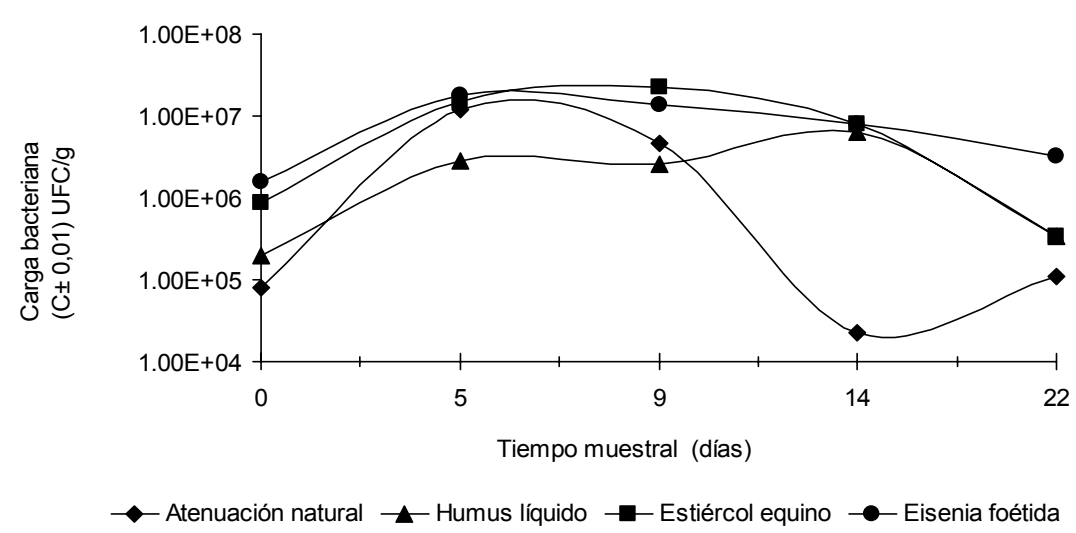

Fig. 2: Evolución de la carga bacteriana mesófila para cada uno de los tratamientos

El hecho por el cual la carga bacteriana mesófila sufre un incremento, se debe que las bacterias autóctonas del suelo se vieron bioestimuladas debido a la adición de estiércol equino, humus líquido y el humus proveniente de la lombriz, los cuales son acondicionadores que aumentan la capacidad de degradación de los microorganismos (Gómez et al., 2006). La figura 3 señala el porcentaje de degradación estimado para cada técnica aplicada con respecto a la evolución de la carga bacteriana.

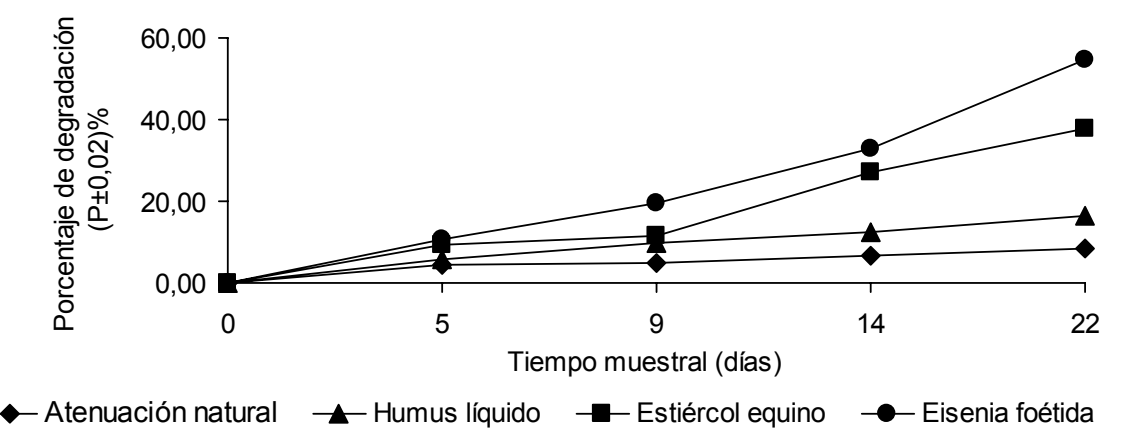

Fig. 3: Porcentaje de degradación de los hidrocarburos totales de petróleo en cada uno de los tratamientos 
Los resultados obtenidos en el suelo control reflejan un porcentaje de degradación de $(8,50 \pm$ $0,02) \%$ al final del tratamiento, que manifiesta una disminución de los hidrocarburos totales de petróleo de $(5,58 \pm 0,02) \%$ a $(5,10 \pm 0,02) \%$, para el estiércol equino hubo un porcentaje de degradación de $(37,72 \pm 0,02) \%$ al final del tratamiento, lo que se traduce en una disminución de los hidrocarburos totales de petróleo de $(5,58 \pm 0,02) \%$ a $(3,47 \pm 0,02) \%$; mientras que para el humus líquido hubo un porcentaje de degradación de $(16,36 \pm 0,02) \%$ al final del tratamiento, con una disminución de los hidrocarburos totales de petróleo de $(5,58 \pm 0,02) \%$ a $(4,66 \pm 0,02) \%$.

En los biorreactores con $E$. foetida la degradación es de $(54,48 \pm 0,02) \%$ al final del tratamiento, lo que resulta en una disminución de los hidrocarburos totales de petróleo de $(5,58 \pm 0,02) \%$ a $(2,54 \pm$ $0,02) \%$, este tratamiento originó los mejores resultados de la carga bacteriana con respecto a los otros tratamientos empleados, esto influye notablemente en la degradación llevada a cabo. A pesar que experimentos anteriores realizados por Schaefer y Filser (2007), describen que la máxima concentración de crudo en suelo a las que pueden ser expuestas los especímenes de $E$. foetida es cercano a los $9500 \mathrm{mg} / \mathrm{kg}$, los resultados obtenidos en la presente investigación permiten comprobar la supervivencia y adaptación de este macroorganismo logrando una degradación de aproximadamente un $55 \%$ en un suelo contaminado con un 5,58 \% (55.800 $\mathrm{mg} / \mathrm{kg})$, es decir, E. foetida toleró una concentración de crudo casi seis veces mayor a la máxima propuesta por Schaefer y Filser (2007), sin una tasa de mortalidad aparente.

En la figura 4 se observan los resultados de las diferentes fracciones del petróleo al inicio y al final del tratamiento en donde no se registraron diferencias significativas $(p>0,05)$ en el tratamiento con humus líquido en la prueba de Tukey HSD ni en la prueba de Dunnett con respecto al tratamiento control, mientras que en los tratamientos con $E$. foetida y estiércol equino si se manifestaron diferencias significativas $(p<0,05)$.

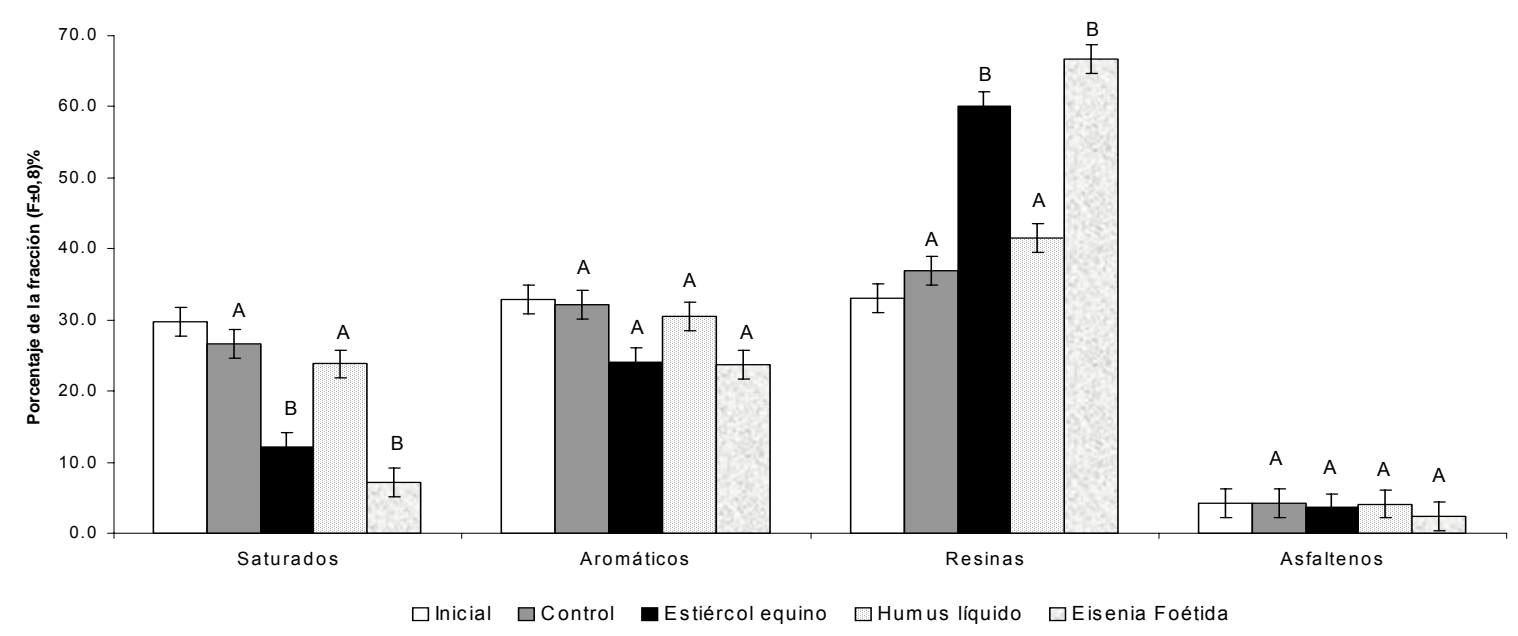

Fig. 4: Porcentaje en peso de las diversas fracciones de crudo al inicio y al final del tratamiento.

(Tratamientos con la misma letra no difieren significativamente, Tukey $p>0,05$ ).

El tratamiento que degradó mayor cantidad de hidrocarburos saturados fue con los ejemplares de $E$. foetida pasando el porcentaje de $(29,8 \pm 0,8) \%$ al inicio, a $(7,2 \pm 0,8) \%$ al finalizar el tratamiento. El segundo tratamiento más efectivo para esta fracción es el tratamiento con estiércol equino con un $(12,3 \pm 0,8) \%$ al final del tratamiento, además puede observarse que el control y el tratamiento con humus líquido, no sufrieron cambios representativos, lo que revela el por qué en dichos biorreactores al finalizar los tiempos muestrales todavía persistía un olor fuerte a petróleo, ya que una de las propiedades físicas de los hidrocarburos saturados es su olor similar al del keroseno (Brown et al., 2004), a diferencia del resto de los biorreactores donde no resaltó esta propiedad. Todo esto es debido a que los microorganismos autóctonos presentes en el suelo les resulta más fácil degradar las fracciones de hidrocarburos saturados (Infante, 2001), ya que éstos constan de ramificaciones lineales de átomos de carbonos (parafinas) y por lo tanto se observan altas tasas de biodegradación de este tipo de hidrocarburos en los tratamientos aplicados. 
Con respecto a la fracción aromática, que inicialmente se encontraba en $(32,9 \pm 0,8) \%$, no se encontraron variaciones significativas $(p=0,0561)$, ni para el tratamiento control, ni para el tratamiento con humus líquido, alcanzándose al final del tratamiento porcentajes de $(32,1 \pm 0,8) \%$ y $(30,6 \pm 0,8) \%$ respectivamente. No obstante para los demás tratamientos, al finalizar el tiempo muestral, se obtuvieron valores de $(24,1 \pm 0,8) \%$ en los biorreactores con estiércol equino y $(23,7 \pm$ $0,8) \%$ en los que contenían macroorganismos de $E$. foetida

Según Infante (2001) la biorremediación permite disminuir significativamente los componentes aromáticos del crudo. Lo cual se verificó en los biorreactores que contenían los especímenes de $E$. foetida, esto es debido a que la lombriz se abre paso a través del suelo excretando humus, que sirve como transporte para que las diversas bacterias entren en contacto de forma más eficaz con el contaminante y se pueda inducir la degradación (Singer et al., 2001).

Otro de los resultados obtenidos fue un aumento progresivo de las fracciones que involucran la cantidad de resinas en todos los tratamientos, correspondiendo al tratamiento con $E$. foetida el valor más alto, alcanzado un $(66,7 \pm 0,8) \%$, al igual que para el estiércol equino de $(60,1 \pm 0,8) \%$. Mientras que el tratamiento con humus líquido alcanzó un valor de $(41,5 \pm 0,8) \%$ y el control un valor de $(36,9 \pm 0,8) \%$. Estos resultados sugieren que hubo ciertos cambios a nivel molecular en la estructura de las resinas producto de las técnicas de biorremediación aplicadas. A pesar de que Infante (2001) señala que las resinas son fracciones recalcitrantes. Álvarez y Guevara (2003), establecen que los microorganismos autóctonos son capaces de poder degradar estos compuestos, ya que durante el proceso de biorremediación no solo actúan las bacterias mesófilas aerobias, sino que también se forman áreas anóxicas en donde crecen bacterias anaeróbicas que son capaces de degradar compuestos de estructura molecular más compleja. La razón del posible aumento es que se trabaja con proporciones expresadas en porcentajes, cuando el valor de alguna fracción disminuye existe un rearreglo de los demás porcentajes, que no involucra si hubo o no un aumento en la masa de la fracción.

En cuanto a la fracción de asfalteno, en la figura 4 se observan todos los cambios a lo largo del biotratamiento, el porcentaje inicial en los biorreactores es de $(4,3 \pm 0,8) \%$ y se constató que esta fracción no presentó casi ningún cambio, salvo en el tratamiento con $E$. foetida $(2,4 \pm 0,8) \%$ al final del tratamiento y $(3,6 \pm 0,8) \%$ para los biorreactores con estiércol equino.

Esto se debe principalmente a que los asfaltenos son fracciones recalcitrantes del crudo ya que son moléculas conjugadas muy complejas que pueden ser capaces de resistir la degradación (Infante, 2001), debido a que los microorganismos son selectivos a la hora de establecer un proceso de degradación. No obstante, se observa un decrecimiento de dicha fracción en los biorreactores que fueron tratados con lombrices, debido principalmente a que las sustancias húmicas tienen un contenido bacteriano que es efectivo en la degradación de compuestos de alta masa molecular como los asfaltenos (Gómez et al., 2006).

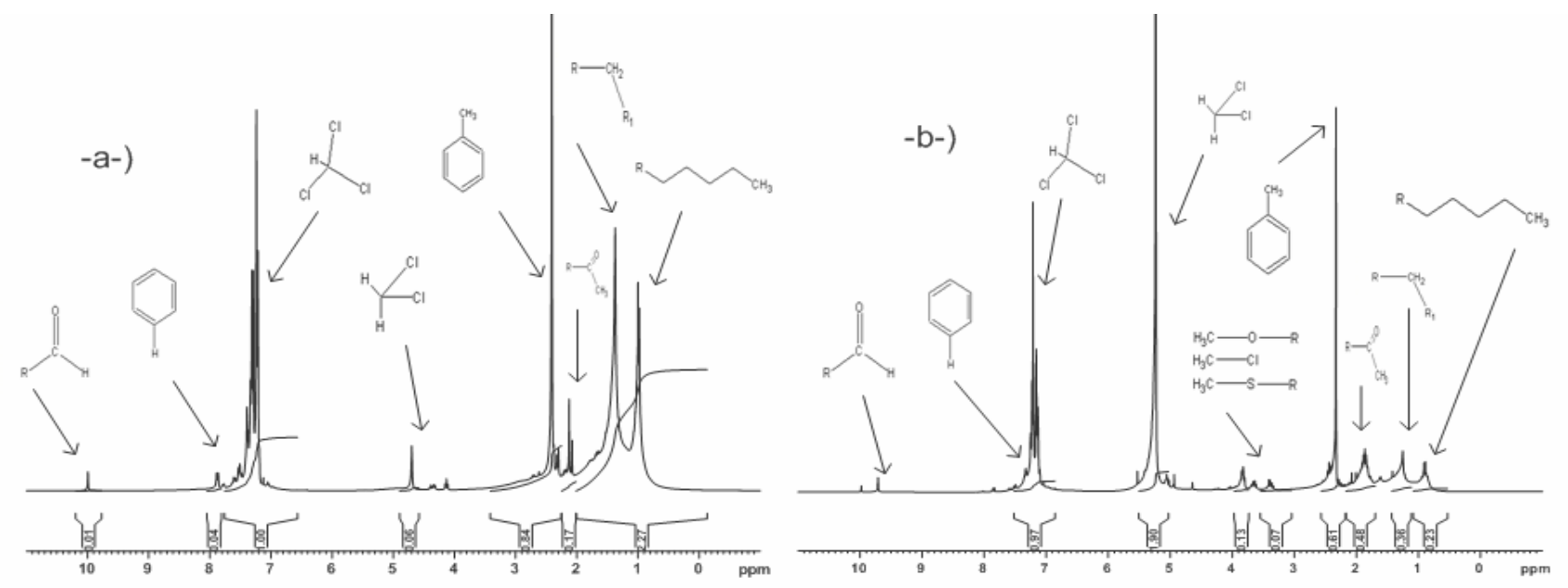

Fig. 5: Espectros de $\mathrm{RMN}^{-1} \mathrm{H}$ para las resinas extraídas en el tratamiento con Eisenia foetida. -a) al inicio de la experimentación. -b) al final de la experimentación 
La figura 5 muestra que no existen muchas diferencias en el espectro, salvo el hecho de que al final del tratamiento aparecen picos entre $(3-4) \mathrm{ppm}$, que reflejan la presencia de hidrógenos unidos a heteroátomos $(\mathrm{N}, \mathrm{O}, \mathrm{S}, \mathrm{Cl})$, producto del proceso de degradación aerobia (oxígeno y nitrógeno) y anaerobia (nitrato, sulfatos y dióxido de carbono) por medio de los microorganismos autóctonos. También se evidencia la presencia de tres sustancias contaminantes (cloroformo, dicloro-metano y tolueno) que se encontraron en las muestras durante la realización de los espectros debido a su pretratamiento.

En el espectro de la figura 6 , se observa la presencia de heteroátomos, esto se debe a que se establecieron condiciones de degradación aerobia que permitieron la inclusión de oxígeno, nitrógeno y azufre en la estructura del asfalteno (Álvarez y Guevara, 2003).

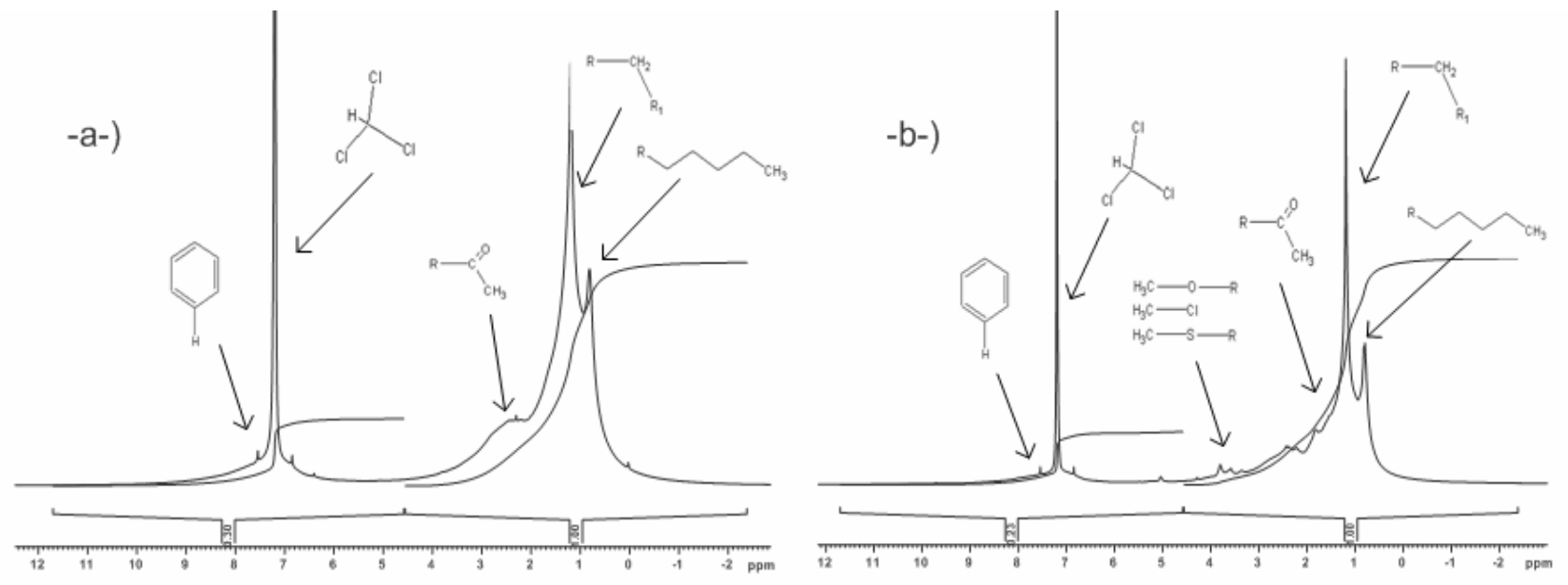

Fig. 6: Espectros de RMN- ${ }^{1} \mathrm{H}$ de asfaltenos extraídos en el tratamiento con Eisenia foetida.

-a) al inicio de la experimentación. -b) al final de la experimentación.

Se evidenció un cambio de coloración de negro a marrón en la fracción de asfaltenos durante el proceso de biodegradación. Debido a que al romperse los enlaces de la parte alifática del asfalteno, este va adquiriendo una estructura más aromática, lo que origina que la luz visible que incide en ellos se absorba en mayor proporción lo que le atribuye como propiedad física una coloración marrón, esto supone un cambio en la estructura del asfalteno producto de la degradación. (Gómez et al., 2006).

\section{CONCLUSIONES}

El suelo utilizado en la experimentación es de textura franco-arenosa con baja cantidad de nutrientes y un alto contenido de acidez. La aplicación de carbonato de calcio en el suelo permitió reducir el contenido de aluminio intercambiable y neutralizar la acidez del suelo, otorgándole una capacidad reguladora de $\mathrm{pH}$. La caracterización del crudo indica que el mismo corresponde a un crudo pesado, mientras que la caracterización del estiércol equino indica que este posee un buen contenido de macronutrientes; no obstante el humus líquido presentó un déficit de los mismos.

Eisenia foetida es un macroorganismo capaz de sobrevivir y tolerar un medio tóxico con condiciones adversas, cercano a $(5,58 \pm 0,02) \%$ de HTP.

El potencial de acidez y el porcentaje de humedad en los biorreactores durante el tratamiento, no sufrieron cambios significativos, según la prueba de Tukey $(p>0,05)$. Los resultados reportaron diferencias significativas $(p<0,05)$ en los tratamientos con los macroorganismos de $E$. foetida y estiércol equino en la degradación HTP, mientras que para el tratamiento con humus líquido no se registraron diferencias significativas en la prueba de Dunnett $(p>0,05)$. Los tratamientos con $E$. foetida y estiércol equino presentaron un alto porcentaje de remoción de hidrocarburos saturados y resinas; mientras que las fracciones de aromáticos y asfaltenos no variaron significativamente $(p>0,05)$. No obstante se verificó la inclusión de heteroátomos dentro de la estructura del asfalteno al final del tratamiento. 


\section{AGRADECIMIENTOS}

A los Laboratorios de Química Orgánica de la Facultad de Ingeniería, al Laboratorio de Investigación de la Facultad Experimental de Ciencia y Tecnología (FACYT) de la Universidad de Carabobo y a la Unidad de Lombricultura de la Facultad de Agronomía de la Universidad Central de Venezuela por su soporte técnico para el desarrollo de este trabajo.

\section{REFERENCIAS}

Álvarez, P. y E. Guevara; "Biorremediación y atenuación natural de acuíferos contaminados por sustancias químicas peligrosas", Consejo de Desarrollo Científico y Humanístico (CDCH-UC) de la Universidad de Carabobo, Valencia, Venezuela (2003).

ASTM D2216, "Determinación del Contenido de Humedad", (en línea), 1998. www.geotecnia.edu.bo/administrador/manual/Humedad.pdf. Acceso: 10 de Agosto (2007).

Brown, T., H. LeMay, E. Bursten y J. Burdge, "Química, la ciencia central", Novena edición, México D.F, Pearson, Prentice Hall (2004).

Callaham, M., A. Stewart, C. Alarcón y S. McMillen; "Effects of earthworm (Eisenia fetida) and wheat (Triticum aestivum) straw additions on selected properties of petroleum-contaminated soils", Environmental Toxicology and Chemistry: 21, 1658-1663 (2002).

Canals, M.; "Biorremediación de suelos contaminados por hidrocarburos: caracterización microbiológica, química y ecotoxicológica”, Tesis Doctoral. Universidad de Barcelona, Facultad de Biología, Departamento de microbiología, Barcelona, España (2005), (en línea) http://www.tdx.cesca.es/TDX-0920105-085623/. Acceso: 30 Agosto (2008).

Casanova, E; Introducción a la ciencia del suelo, Consejo de Desarrollo Científico y Humanístico (CDCH-UCV) de la Universidad central de Venezuela, Caracas, Venezuela (2005).

Contreras S., D. Álvarez, L. Dendooven; "Removal of polycyclic aromatic hydrocarbons from soil amended with biosolid or vermicompost in the presence of earthworms (Eisenia fetida)" Soil Biology and Biochemistry: 40,1954-1959 (2008).

COVENIN 902; Comisión Venezolana de normas industriales. Método para recuento de colonias de bacterias aerobias en placas de Petri. $2^{\text {da }}$ revisión (1987).

Decreto 2.635, República de Venezuela, Reforma parcial del decreto contentivo de las normas para el Control de la Recuperación de Materiales Peligrosos y el manejo de desechos peligrosos. Gaceta Oficial de la República de Venezuela Nº 5.245 Extraordinaria. Caracas (1998).

Durán L. y C. Henríquez; "Caracterización química, física y microbiológica de vermicompostes producidos a partir de cinco sustratos orgánicos". Revista Agronomía Castrense: 31, 001, 41-51 (2007) (en línea) http://redalyc.uaemex.mx/redalyc/pdf/436/43631105.pdf. Acceso: 31 Agosto (2008). Ferruzzi, C; Manual de lombricultura, Ediciones MUNDI- PRENSA, Madrid, España (1994).

Gallego, P., R. Cuéllar y J. Dussán; "Biorremediación de residuos de petróleo", (en línea), 2004. http://www.mindefensa.gov.co/derecho/mea01gua.html. Acceso: 16 Enero (2008).

Geissen, V. y otros seis autores; "Using earthworms to test the efficiency of remediation of oilpolluted soil in tropical Mexico", Ecotoxicology and Environmental Safety: 70, Issue 1 (2008).

Gómez S. E., y otros siete autores; "Biodegradación de asfaltenos del Prestige mediante la aplicación de técnicas de compostaje-vermicompostaje". Universidad Complutense Madrid. Revista Residuos: 16 (92), 56-63 (2006).

Gutiérrez, H. y R. De la Vara; “Análisis y diseño de experimentos” Primera edición. México. McGrawHill (2004). 
Hubálek T. y otros cuatro autores; "Ecotoxicity monitoring of Hydrocarbon-Contaminated Soil during bioremediation: A case of study". Arch. Environ. Contam. Toxicol.: 52, 1-7 (2007).

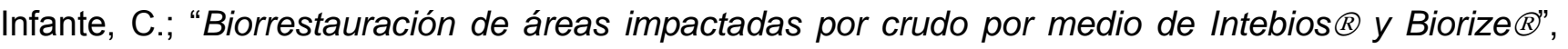
Interciencia: 26, 504-507 (2001)

La Grega, M., P. Buckingham y J. Evans; "Gestión de residuos tóxicos. Tratamiento, eliminación y recuperación de suelos", Volumen II. Editorial Mc.Graw Hill, Madrid, España (2002).

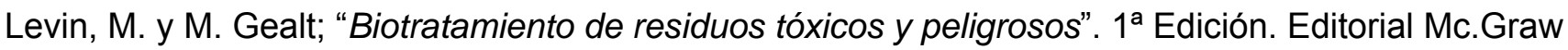
Hill, Madrid (1997).

Monard C. y otros cinco autores; "Combined effect of bioaugmentation and bioturbation on atrazine degradation in soil". Soil Biology and Biochemistry: 40, 2253-2259 (2008)

Rodríguez, R., J. Moreno y M. Larreal; "Comportamiento de la conductividad eléctrica en dos series de suelo del sector Caño San Miguel, municipio Mara, estado Zulia durante un período de dos años". Universidad del Zulia. Revista Facultad de Agronomía: 23 (4) 395-406 (2006).

Sandoval, J; "Acondicionadores y mejoradores de suelo". Instituto colombiano agropecuario. Medellín. Colombia (2002).

Schaefer, M. y J. Filser; "The influence of earthworms and organic additives on the biodegradation of oil contaminated soil". Applied Soil Ecology: 36, 53-62 (2007).

Singer, A.C., y otros cuatro autores; "Contribution of earthworms to PCB bioremediation". Soil Biology \& Biochemistry, 33, 765-776 (2001).

Statistix 8.0 for Windows, "Analytical Software”. Tallahassee, FL (2003).

TMECC, Test Methods for the Examination of Composting and Compost. U.S. The Composting Council Research and Education Foundation (2001).

US EPA Method 3540C; Agencia de protección ambiental (EPA), Soxhlet Extraction. Determinación de HTP (1996).

US EPA (8020/8100); Agencia de protección ambiental (EPA), Caracterización de crudos en fracciones SARA (1986).

Vívas E., B. Bravo y G. Chávez; "Estudio de las fracciones ácidas y básicas extraídas de asfaltenos de un crudo pesado". Revista Ciencia: 10 (4) 419-428 (2002) 